\title{
Síndrome de Chediak-Higashi en fase acelerada
} Reporte de un caso

\author{
Gallegos-Cisneros Edmundo*, Cardiel-Silva Maricela**, Peral-Ibarra Roma**, Tavares-Rodríguez \\ Edgar Jesús****, Ávila-Chávez Abdon ${ }^{* * * * *}$
}

\begin{tabular}{|c|c|}
\hline $\begin{array}{l}\text { Resumen } \\
\text { Introducción: El síndrome de Chediak-Higashi (SCH) es un pa- } \\
\text { decimiento congénito raro. Se han reportado poco menos de } \\
500 \text { casos en los últimos } 20 \text { años en todo el mundo. Es una } \\
\text { enfermedad que se transmite de manera autonómica recesi- } \\
\text { va; se manifiesta en la infancia temprana por albinismo oculo- } \\
\text { cutáneo y pelo plateado, con infecciones bacterianas y virales } \\
\text { por inmunodeficiencia, secundario a alteraciones estructurales } \\
\text { y funcionales de los leucocitos; las particularidades estructura- } \\
\text { les pueden identificarse al microscopio en un frotis de sangre } \\
\text { periférica. Caso clínico: Es un preescolar eutrófico de } 4 \text { años } \\
\text { de edad que debuta con gran hepato-esplenomegalia y fiebre; } \\
\text { en la exploración se observó la presencia de pelo plateado, } \\
\text { los padres negaron infecciones recurrentes. Se realizaron los } \\
\text { estudios pertinentes observándose pancitopenia, estudio de } \\
\text { cabello con cúmulos melánicos pequeños y desordenados, } \\
\text { grandes granulaciones intracelulares en polimorfonucleares } \\
\text { y linfocitos; inmunofenotipo negativo para células malignas e } \\
\text { incremento considerable en niveles de colesterol, triglicéridos, } \\
\text { inmunoglobulinas IgG totales y transferrina con lo que se lle- } \\
\text { ga al diagnóstico de síndrome de Chediak Higashi. Se inició } \\
\text { tratamiento con ciclosporina, inmunoglobulina G y prednisona, } \\
\text { lamentablemente por su evolución tan avanzada cursa con } \\
\text { estado de choque con muy malas esperanzas de vida. LUXMÉ- } \\
\text { DICA, AÑo 10, NúmER031, SEPTIEMBRE-DICIEMBRE 2015, PP 61-70 }\end{array}$ & $\begin{array}{l}\text { Abstract } \\
\text { Ilntroduction: The Chediak-Higashi syndrome (CHS) is a rare } \\
\text { congenital condition; slightly less than } 500 \text { cases have been } \\
\text { reported in the last } 20 \text { years all over the world. It is a disease } \\
\text { that is transmitted from autonomic recessive manner; mani- } \\
\text { fests itself in early childhood by albinism oculocutaneous and } \\
\text { silver hair, with bacterial and viral infections by immunode- } \\
\text { ficiency, secondary to structural and functional alterations of } \\
\text { leukocytes; the structural particularities can be identified under } \\
\text { the microscope in a peripheral blood smear. Clinical case: it is } \\
\text { a Eutrophic preschool for 4-year-old who made his debut with } \\
\text { great hepato-splenomegaly and fever was observed in the cli- } \\
\text { nical examination the presence of silver hair, parents do not } \\
\text { refer to recurrent infections, the relevant studies are performed } \\
\text { with pancytopenia, study of hair with melanistic small and clut- } \\
\text { tered clusters, large intracellular granulations in polymorpho- } \\
\text { nuclear and lymphocytes, negative immune to malignant cells, } \\
\text { significant increase in levels of cholesterol, triglycerides, total } \\
\text { IgG immunoglobulins and transferrin with what it comes to } \\
\text { the diagnosis of Chediak Higashi syndrome began treatment } \\
\text { with Cyclosporine, immunoglobulin G and prednisone unfortu- } \\
\text { nately by its evolution so advanced, courses with shock with } \\
\text { extremely poor life expectancy. LUX MÉDICA, AÑO 10, NúMERO } \\
\mathbf{3 1 , S E P T I E M B R E - D I C I E M B R E ~ 2 0 1 5 , ~ P P ~} \mathbf{6 1 - 7 0}\end{array}$ \\
\hline $\begin{array}{r}\text { Palabras cla } \\
\text { bu }\end{array}$ & mogopumin, neutropmi \\
\hline
\end{tabular}

\footnotetext{
Médico Pediatra Adscrito al Hospital General de Zona Número 1 del Instituto Mexicano del Seguro Social de Aguascalientes.

** Médico Hematólogo Adscrito al Hospital General de Zona Número 1 del Instituto Mexicano del Seguro Social de Aguascalientes.

*** Médico Residente de Pediatría Hospital General de Zona Número 1 del Instituto Mexicano del Seguro Social de Aguascalientes.

**** Médico Interno de Pregrado del Instituto Mexicano del Seguro Social de Aguascalientes.

***** Médico Residente de la especialidad de Medicina Interna del Instituto Mexicano del Seguro Social de Aguascalientes.
}

Fecha de recibido: 30 de septiembre de 2015

Fecha de aceptación: 30 de octubre 2015

Correspondencia: Dr Edmundo Gallegos Cisneros Servicio de Pediatría. Hospital General de Zona Número 1. Instituto Mexicano del Seguro Social. Avenida José María Chávez 1202, Colonia Lindavista. Código Postal 20270 Aguascalientes, Aguascalientes, México. Teléfono: +01 (449) 9159050 Correo electrónico ejtr_2206@live.com 


\section{Introducción}

El síndrome de Chediak-Higashi $(\mathrm{SCH})$ es una deficiencia inmune de carácter autosómico recesivo, que se origina por mutaciones del gen 1q42 que codifica la proteína lisosome traffcking regulator (LYST); esta proteína se encarga de la regulación del transporte lisosomal y la función del citoesqueleto ${ }^{1-4}$. Este defecto impide la formación normal de los fagolisosomas y de los melanosomas, vesículas primordiales en el proceso de fagocitosis, ${ }^{5}$ lo que afecta la función de varios sistemas orgánicos, especialmente la función de los leucocitos.

Si bien en 1943 y en 1948 aparecen las primeras descripciones clínicas, corresponde a Chediak (1952) y a Higashi (1954) hacer la descripción con la que hasta ahora se le distingue: albinismo oculocutáneo, infecciones recurrentes por alteraciones lisosómicas y citoesqueléticas, principalmente en las siguientes células: neutrófilos, monocitos, hepatocitos, células de los túbulos renales. ${ }^{5}$

Las características clínicas que distinguen a estos pacientes son hipopigmentación de la piel, ojos y pelo que adquiere un color plateado; las infecciones en ellos son frecuentes especialmente por $\mathrm{S}$. aureus, estreptococos, aspergillus y cándida. En el frotis de sangre periférica se observan células sanguíneas anormales: neutrófilos con gránulos gigantes basófilos, linfocitos vacuolados con gránulos eosinofílicos y plaquetas con gránulos gigantes. Puede encontrarse hepato-esplenomegalia, equimosis de fácil aparición en la piel y neuropatía periférica. El examen microscópico del cabello permite apreciar acúmulo anormal de melanina. ${ }^{3,5}$ En la actualidad, se acepta la existencia de tres presentaciones clínicas.

La forma más frecuente y grave es la denominada infantil, que supone aproximadamente un $85 \%$ de los casos y que cursa con un espectro clásico de síntomas y signos oculocutáneos, cuadros infecciosos y diátesis hemorrágica. En su evolución natural, se han descrito dos fases: una de buena tolerancia y otra fase acelerada ( $\mathrm{SCHA}$ ) que se caracteriza por fotofobia, nistagmos, esplenomegalia, adenopatías por infiltración linfohistiocítica en diversos órganos y estados depresivos. La tomografía axial computarizada (TAC) y la resonancia magnética nuclear (RMN) muestran cambios atróficos del cerebro.

En el electroencefalograma (EEG) se han reportado en algunos casos anormalidades paroxísticas. La electromiografía (EMG) muestra en ciertos pacientes retardo en la conducción nerviosa. ${ }^{6,7}$ se ha asociado con infecciones por virus de Epstein bar en el 70\% de los casos. Se ha descrito una forma adolescente que cursa con hipopigmentación, infecciones recurrentes, pero no graves, y un discreto retraso en el desarrollo. Estos pacientes no presentan fase SCHA. Finalmente, la forma adulta cursa con sintomatología neurológica de gravedad variable como ataxia, convulsiones y, excepcionalmente, con datos de infección. 
Los hallazgos de inmunodeficiencias descritos hasta la actualidad son: anormalidades en los lisosomas con incapacidad para liberar su contenido durante la fagocitosis. Defecto en la función bactericida intracelular por disfunción de las células killer (NK) ${ }^{6,7}$. El examen microscopio del cabello permite observar un acúmulo anormal de mielina ${ }^{8-9}$.

En cuanto a la fisiopatogenia se ha documentado la presencia de un defecto en las proteínas de trasporte intracelular. La enfermedad se debe a una deficiencia de LYST localizado en el gen 1q42-43 que codifica una proteína de unos 3801 aminoácidos ${ }^{10}$. Esta proteína es muy importante para la regulación del trasporte lisosomal y la función del citoesqueleto ${ }^{11}$, Expresada en el citoplasma de las células de diversos tejidos produce anormalidades en el tránsito de proteínas en los organelos, alterando la síntesis, mantenimiento y almacenamiento de los gránulos secretorios de varios tipos de células ${ }^{12}$. Este defecto impide la formación normal de los fagolisosomas y de los melanosomas, vesículas que intervienen en el proceso de fagocitosis ${ }^{12}$.

Los lisosomas de los leucocitos y fibroblastos, las plaquetas, gránulos azúrofilos en los neutrófilos, melanosomas y meloncitos, células foliculares tiroideas y tubulares renales presentan alteraciones morfológicas ${ }^{12,13}$, lo que indica un camino común en la síntesis de los organelos responsables de los depósitos, característica principal del SCH que dificultan la función de éstos ${ }^{14}$.

Otros autores señalan que en la fase acelerada de la enfermedad hay un defecto de la vía CTLA-415, además de la activación de linfocitos T CD28 unidos a B7.1 (CD80) y B 7.2 (CD86) de células presentadoras del antígeno CTLA-4 o CD152 que se unen a los B7.1 Y B7.2. A diferencia de los CD28, estas moléculas envían señales para la inhibición de los linfocitos $T$ para que no se expresen en la superficie celular, éstos, al no ser regulados, evolucionan a un síndrome linfoproliferativo con leucopenia y trombocitopenia ${ }^{16}$.

\section{Presentación del caso clínico}

Una preescolar eutrófica de 4 años de edad, fue remitida al servicio de urgencias pediátricas del Hospital General de Zona \#1 de Aguascalientes, nacida en Tepetate, municipio de Loreto Zacatecas. El motivo de consulta fue fiebre, pancitopenia y hepato-esplenomegalia. Es producto del primer embarazo de madre de 27 años, la cual debido a que padece artritis reumatoide es tratada con metotrexate, prednisona y ciclosporina, fármacos que ingirió dentro del primer trimestre del embarazo. No hay historia de consanguinidad.

Inicia con su padecimiento 30 días previos a su ingreso cuando le establecen el diagnóstico de síndrome linfoproliferativo e iniciaron tratamiento con prednisona; 
en estudio por hematología pediátrica se transfundió en tres ocasiones concentrados plaquetarios, sin resultados satisfactorio por lo que se realiza aspirado de médula ósea e inmunofenotipo, el cual descarta proceso linfoproliferativo. En frotis de sangre periférica no se encontraron datos de malignidad. Ingresó y egresó en cuatro ocasiones para transfusión de plaquetas, y se da de alta del servicio de hematología descartado el síndrome linfoproliferativo.

Posteriormente ingresa al servicio de pediatría para su estudio donde encuentran a una paciente en regulares condiciones generales, piel grisácea en cara, cuero cabelludo, tronco y extremidades; en el fondo de ojo se observa palidez de retina acompañada de fotofobia, iris de aspecto normal. Al evaluar el cabello, éste presentó textura delgada y frágil, con brillo metálico (figura 1A, 1B). La cavidad oral se encontró normal, a la palpación abdominal se encontró borde hepático a seis $\mathrm{cm}$ por debajo del reborde costal y el bazo a la altura de la cresta iliaca anterosuperior y en la extremidades inferiores se encontró edema bimaleolar. La tomografía axial computada (TAC) reportó gran hepato esplenomegalia, así como crecimiento ganglionar peripancreático (figura 1B).

La radiografía de tórax no muestra datos de infiltrados pulmonares. El frotis sanguíneo mostró neutrófilos con gránulos gigantes, vacuolas intracitoplasmáticas en linfocitos y gránulos eosinófilicos. En médula ósea se observaron linfocitos con múltiples vacuolas, niveles anormalmente bajos de plaquetas, anemia normocítica hipocrómica, signos hematológicos característicos del síndrome (figura 2). En el examen microscópico de cabello mostro pequeño cúmulos desordenados, regulares de melanina dispuesta de manera anormal (figura 3).

Se inició tratamiento con prednisona intravenosa $(1 \mathrm{mg} / \mathrm{kg} /$ día), gammaglobulina $(1 \mathrm{mg} / \mathrm{kg} /$ día), ciclosporina $(5 \mathrm{mg} / \mathrm{kg} /$ día) y cefotaxima $(50 \mathrm{mg} / \mathrm{kg} /$ día). Se refiere a tercer nivel de atención para tratamiento especializado. El día 27 de julio el paciente cae en estado de choque séptico, con deterioro generalizado.

\section{Discusión}

El síndrome hemofagocítico se caracteriza por fiebre mayor a 38.3 grados centígrados, esplenomegalia mínimo de $3 \mathrm{~cm}$, hemoglobina menor a $9 \mathrm{gr} / \mathrm{dL}$, trombocitopenia menor a 100,000 plaquetas $/ \mathrm{mm}^{3}$, leucopenia/neutropenia menor a 1000 células $/ \mathrm{mm}^{3}$, hipertrigliceridemia, hipofibrinogenemia, hemofagocitosis en médula ósea sin evidencia de hipoplasia o neoplasia maligna ${ }^{17-19}$. En algunas patologías que integran este conjunto sindromático se agregan datos cardinales y altamente diferenciables como el cabello plateado, los datos histopatológicos de cabello o los hallazgos del frotis sanguíneo.

Las diferentes etiologías ligadas al síndrome hemofagocítico están ligadas entre sí por tener una base fisiopatológica similar, el diagnóstico diferencial se logra en función de datos clínicos histopatológicos y de biología molecular, las principales entidades se enlistan en la tabla 2.

Ciertamente el estudio molecular es el estándar de oro en este síndrome, sin embargo los parámetros clínicos y bioquímicos son el otro pilar importante para la correcta discriminación entre las mismas. 
Ante un paciente con datos clínicos sugestivo de este síndrome es importante poder realizar el abordaje teniendo como diagnósticos diferenciales las patologías integrantes del síndrome hemofagocítico que se resumen en la tabla $3^{20-22}$.

En el abordaje inicial de nuestro paciente se encontraba en la edad con la cual se debutan los síntomas y signos de la fase acelerada; la fiebre, el ataque al estado general y la hepatoesplenomegalia fueron los principales síntomas de consulta, además mostró todos los signos bioquímicos, en contraste con el síndrome de Griselli tipo 2 (SG2) se encontraron granulaciones anormales las cuales no son parte de la clínica de SG2, comparando el caso con una linfohistiocitosis hemofagocitico familiar (FHL3), en nuestro caso encontramos hipopigmentación del cabello así como color gris plateado, granulaciones anómalas en sangre y en médula ósea lo cual no se encuentran en este síndrome.

\section{Conclusiones}

Las consideraciones terapéuticas actuales no ofrecen una gran esperanza de vida, hablamos de sesiones agresivas de quimioterapia que paradójicamente comprometerían aún más la respuesta inmunológica, seguido de trasplantes de médula ósea que ayudarían en fase acelerada, sin embargo aun así la esperanza de vida es muy poca, se ha considerado la esplenectomía en casos de sangrados masivos, lamentablemente la evaluación costo beneficio no ha mostrado grandes aportes hacia la evolución del paciente ${ }^{22-24}$. El uso de antimicrobianos a grandes dosis comprometen aún más la función hepática y precisamente estas alteraciones aunadas a la inmunosupresión, llevan a los pacientes a desarrollar falla orgánica múltiple y morir. Actualmente la sobrevivencia de los enfermos con este síndrome sigue siendo mínima; sin embargo el diagnóstico temprano puede mejorar el pronóstico y la calidad de vida de estos pacientes.

\section{Bibliografía}

1. Chediak, M.: "Nouvelle anomalie leucocytaire de caractere constitutionnel et familial ». Rev. Hemat. 7: 362-367, 1952. PubMed ID: 130045533.

2. De Beer, H. A.; Anderson, R.; Findlay, G. H.:" Chediak-Higashi syndrome in a 'black' child: clinical features, immunological studies, and optics of the hair and skin". S. Afr. Med. J. 60: 108-112, 1981. PubMed ID: 7256443

3. Donohue, W. L.; Bain, H. W.: "Chediak-Higashi syndrome, a lethal familial disease with anomalous inclusions in theleukocytes and constitutional stigmata: report of a case with necropsy". Pediatrics 20: 416-430, 1957. PubMed ID: 13465231

4. Dufourcq-Lagelouse, R.; Lambert, N.; Duval, M.; Viot, G.; Vilmer, E.; Fischer, A.; Prieur, M.; de Saint Basile, G.: "Chediak-Higashi syndrome associated with maternal uniparentalisodisomy of chromosome 1 ». Europ. J. Hum. Genet. 7: 633-637, 1999. Pub-
Med ID: 10482950

5. Abo, T.; Roder, J. C.; Abo, W.; Cooper, M. D.; Balch, C. M.: "Natural killer (HNK-1+) cells in ChediakHigashi patients are present in normal numbers but are abnormal in function and morphology". J. Clin. Invest. 70: 193-197, 1982. PubMed ID: 7085883

6. Haddad $E$, Le Deist $F$, Blanche $S$, Benkerrou $M$, Rohrlich $\mathrm{P}$, Vilmer $\mathrm{E}$, et al.. "Treatment of ChediakHigashi syndrome by allogeneic bonemarrow transplantation: report of 10 cases". Blood 1995; 85: 3.328-3.333. 34. Misra, V. P.; King, R. H. M.; Harding, A. E.; Muddle, J. R.; Thomas, P. K.: "Peripheralneuropathy in the Chediak-Higashi syndrome". Acta Neuropath. 81: 354-358, 1991. PubMed ID: 2058369

7. Uyama, E.; Hirano, T.; Ito, K.; Nakashima, H.; Sugimoto, M.; Naito, M.; Uchino, M.; Ando, M.: "Adult Chediak-Higashi syndrome presenting as parkinsonism and dementia". Acta Neurol. Scand. 89: 175183, 1994. PubMed ID: 8030398 
8. Luis Montiel López, Juan Gabriel Posadas Calleja, Guillermo Domínguez Cherit "Fisiopatología del síndrome hemofagocítico (linfohistiocitosis hemofagocítica) Artículo de revisión" MedIntMex 2005;21:7581

9. Norberto Sotelo-Cruz, Gilberto Covarrubias-Espinoza, Rebeca Gómez-Rivera, María José García "Chediak-Higashi syndrome in aninfant. A case in acceleratedphase" Revista Mexicana de Pediatría 2007; 74(3):113-118.

10. Ayas $\mathrm{M}$, Al-Ghonaium A. In patientswith ChediakHigashi syndrome undergoingallogeneic SCT, does adding etoposide to the condition ingregimenim prove the out come? BoneMarrow Transplant. 2007; 40:603.

11. Diagnóstico y tratamiento Sindrome de Chediak-Higashi, Chediak-Higashi síndrome Francisco Jose Ortunoa, Jose Luis Fusterb y Andres Jereza / MedClin (Barc). 2010;135 (11):512-518.

12. Aspectos psiconeuroinmunológicos del Síndrome de Chediak-Higashi: artículo de revisión Teraiza E. Mesa Rodríguez, Alcmeon, Revista Argentina de Clínica Neuropsiquiátrica, 2008;14(4):66-82.

13. Síndrome de Chediak Higashi. Reporte de un Caso Roba Izzeddin, Maria Julia Salas, Armida Acuña, Rula Izzeddin 1Departamento Prostodoncia y Oclusión, Facultad de Odontología. Escuela de Medicina. Escuela de Bionalisis, Facultad de Ciencias de la Salud. Universidad de Carabobo Vol. IX No. 1, Enero - Junio 2008

14. Los trastornos de la secreción lisosomal en la sinapsis inmune y otros tejidos I. Jiménez Garcíaa, A. Galera Minarroa, E. Llinares Riestraa, M. Bermúdez Cortésa, S. Alfayate Miguélezb y J.L. Fuster Solera AnPediatr (Barc). 2012;76(2):92-97

15. Dell'Angelica EC, Mullins C, Caplan S, Bonifacino JS. Lysosome related organelles. FASEB J. 2000;14:1265---78. 2. Huizing M, Helip-Wooley A,
Westbroek W, Gunay-Aygun M, Gahl WA. Disorders of Lysosome-relatedOrganelleBiogenesis: Clinical and Molecular Genetics. Annu Rev Genomics Hum Genet. 2008;9:359-386.

16. Raposo G, Marks MS, Cutler DF. Lysosome-RelatedOrganelles: Driving post-Golgi compartment sinto specialisation. CurrOpinCell Biol. 2007;19:394-401. 4. ZurStadt U, Beutel K, Kolberg S, Schneppenheim $R$, Kabisch $H$, Janka $G$, et al. Mutation spectrum in children with primary hemophagocyticlymphohistiocytosis: molecular and functional analyses of PRF1, UNC13D, STX11, and RAB27A. HumMutat. 2006;27:62-68.

17. Stinchcombe JC, Majorovits E, Bossi G, Fuller $S$, Griffiths GM. Centrosome polarization delivers secretory granules to the immunologicalsynapse. $\mathrm{Na}$ ture. 2006;443:462.

18. Lieberman J. TheABCs of granule-mediated cytotoxicity: new weapons in the arsenal. NatRevlmmunol. 2003;3: 370.

19. Fischer A, Latour S, De Saint Basile G. Genetic defects affecting lymphocytecytotoxicity. CurrOpinImmunol. 2007;19:348

20. Janka GE, Schneider EM. Modern management of children with haemophagocyticlymphohisitocytosis. Br J Haematol. 2004;124.

21. Stinchcombe J, Bossi G, Griffiths GM. Linkingalbinism and immunity: the secrets of secretory lysosomes. Science. 2004;305:55

22. Kaplan J, De Domenico I, McVey Ward D. Chediak Higashi Syndrome. CurrOpinHematol. 2008;15:22

23. Holt OJ, Gallo F, Griffiths GM. Regulating secretory lysosomes. J Biochem. 2006;140

24. Ménasché G, Pastural E, Feldmann J, Certain S, Ersoy $F$, Dupuis $S$, et al. Mutations in RAB27A cause Griscelli syndrome associatedwithhemophagocytic syndrome. NatGenet. 2000;25:173.

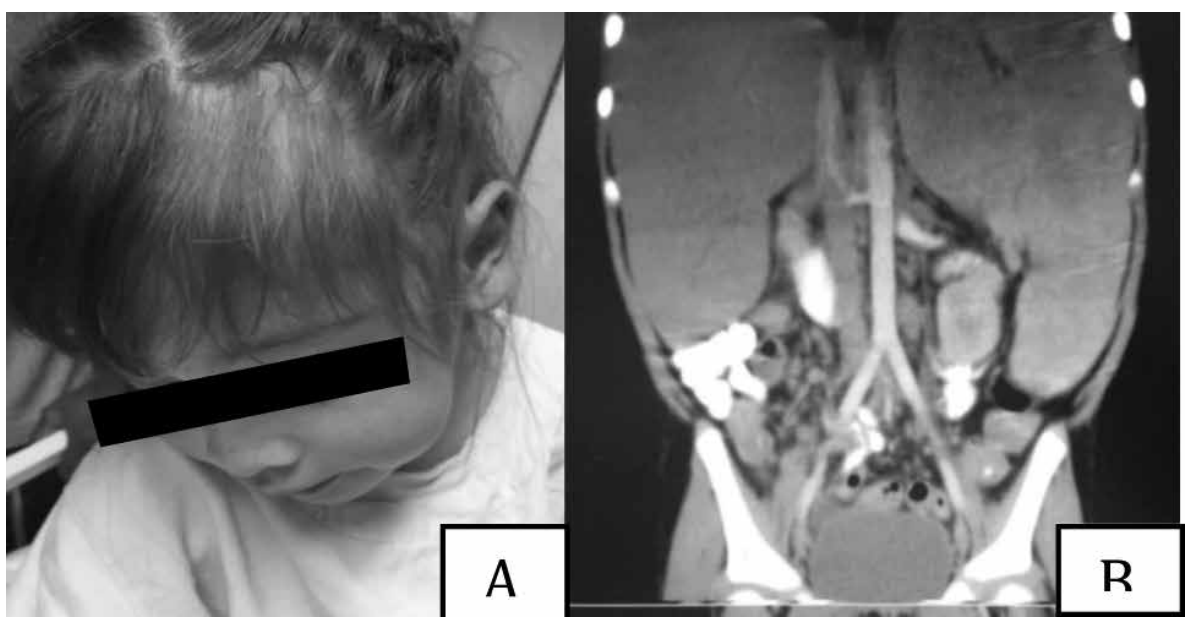

Fig. 1 A.- Preescolar eutrófico con brillo metálico en el cabello. Fig. 1B Corte Coronal de Tomografía axial simple con gran hepatoesplenomegalia. 

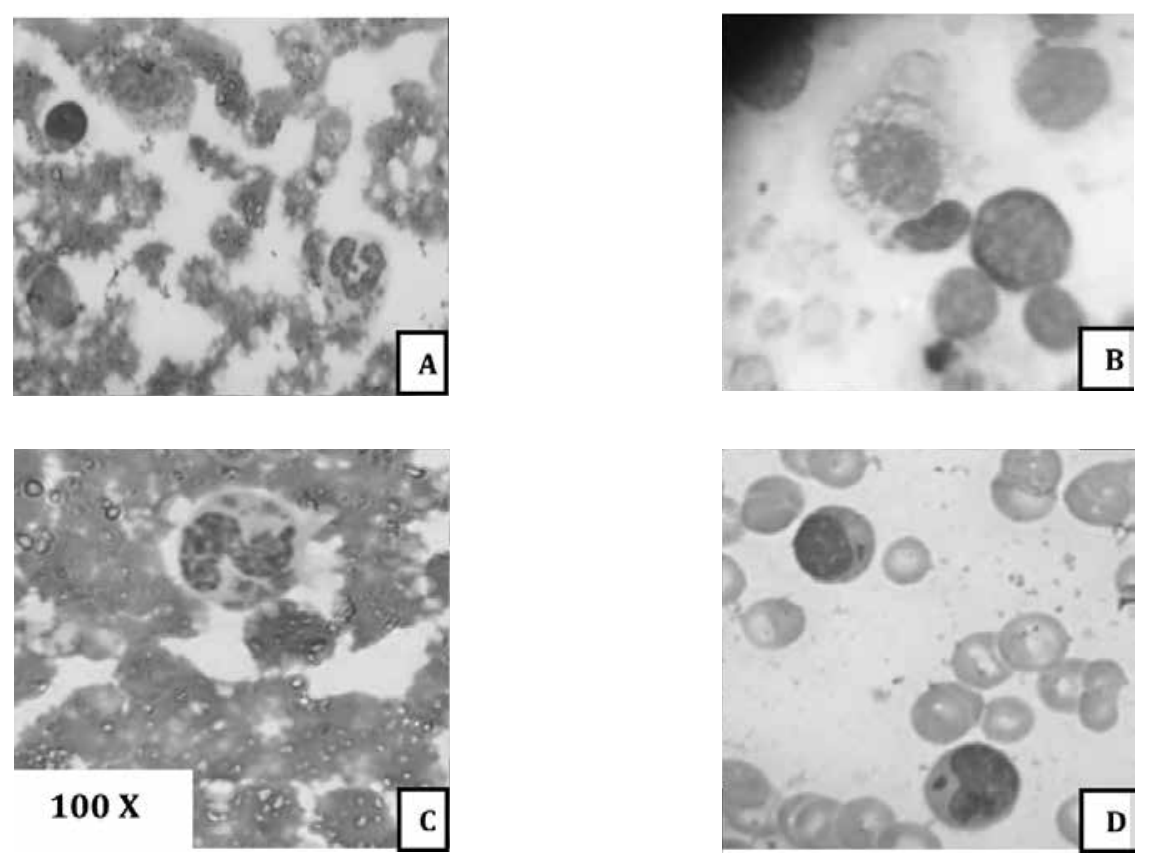

Fig. 2.-A) linfocito con gran acúmulo de gránulos y neutrófilo con gránulos gigantes intracitoplásmicos B) aspirado de médula con múltiples vacuolas intracitoplásmicas en linfocitos, C) neutrófilo con gránulos gigantes intracitoplásmicos, D) linfocitos de sangre periférica con gránulo único.

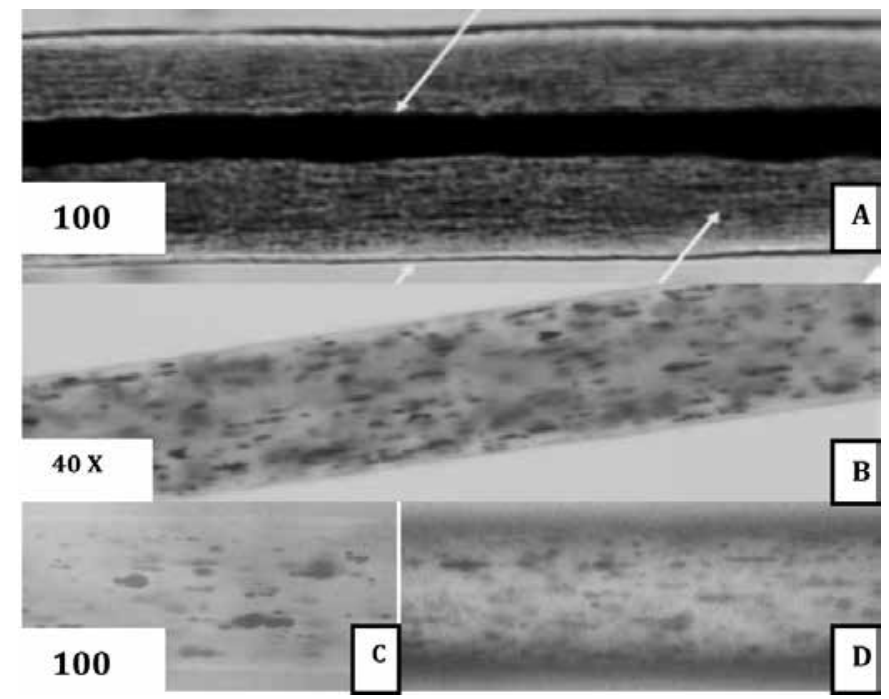

Fig. 3 A Se observa imagen microscópica de un cabello normal, B) Imagen del cabello de la paciente a 40X que muestra gránulos pequeños y desordenados de pigmento melánico, $C, D$ ) Imagen que visualiza a detalle los cúmulos de pigmento melánico. 


\section{Tabla |}

\section{RESULTADOS DE LABORATORIO}

\section{HEMATOLOGIA/COAGULACION}

\begin{tabular}{|c|c|c|c|c|c|c|c|}
\hline & $01 / 06 / 2015$ & $07 / 07 / 15$ & $10 / 07 / 15$ & $20 / 07 / 15$ & $21 / 07 / 15$ & $23 / 07 / 15$ & $24 / 07 / 15$ \\
\hline Hemoglobina (gr/dl) & 7.6 & 8.4 & 8.1 & 9.7 & 9.2 & 6.9 & 9.1 \\
\hline Leucocitos (mm3) & 4870 & 4000 & 2950 & 4800 & 4600 & 2840 & 3690 \\
\hline Neutrófilos (\%) & 2.7 & 2.4 & 8.3 & 8.5 & 5.3 & 9.6 & 7.5 \\
\hline Linfocitos (\%) & 82.7 & 86 & 77.5 & 84 & 89 & 82.6 & 81 \\
\hline Hematocrito $(\%)$ & 25.7 & 26 & 25.4 & 29.9 & 27.7 & 22.1 & 30 \\
\hline Hierro Sérico & & & & & & & 446 \\
\hline VSG (mm/hr) & 30.0 & & & & & & 54 \\
\hline Plaquetas(cel/mm3) & 20000 & 22000 & & 18000 & 20000 & 28000 & 30000 \\
\hline TP (segundos) & & & & 14 & & & 11 \\
\hline TTPA (segundos) & & & & 45 & & & 30 \\
\hline Reticulocitos (\%) & & & & & & 0.9 & \\
\hline \multicolumn{8}{|l|}{ QUIMICA SANGUINEA } \\
\hline Glucosa (mg/dl) & 68 & & & 64 & & 65 & 79 \\
\hline Urea $(\mathrm{mg} / \mathrm{dl})$ & & & & 53 & & 29 & \\
\hline Creatinina (mg/dl) & 0.4 & & & 0.7 & & 0.6 & 0.3 \\
\hline Amonio (mg/dl) & & & & & & 66 & \\
\hline Bilirrubina $\mathrm{D}(\mathrm{mg} / \mathrm{dl})$ & & & & 1.5 & & & 1.5 \\
\hline Bilirrubina I (mg/dl) & & & & 1.0 & & & 1.0 \\
\hline Bilirrubina $\mathrm{T}(\mathrm{mg} / \mathrm{dl})$ & & & & 2.5 & & & 2.5 \\
\hline $\mathrm{TGO}(\mathrm{U} / \mathrm{L})$ & & 148 & & 474 & & & 269 \\
\hline $\mathrm{TGP}(\mathrm{U} / \mathrm{L})$ & & 74 & & 190 & & & 110 \\
\hline $\mathrm{LDH}(\mathrm{U} / \mathrm{L})$ & & 2146 & & 1771 & & & 1100 \\
\hline Albumina Gr/l & & 2.4 & & & & & 2.8 \\
\hline Ácido úrico (mg/dl) & & & & & & 4.8 & 2.0 \\
\hline F. Alcalina (U/L) & & 919 & & & & & 390 \\
\hline Fibrinógeno(mg/dl) & & & & & & 188 & \\
\hline Ferritina $(\mathrm{mg} / \mathrm{dl})$ & & & & & & & 1900 \\
\hline Triglicéridos(mg/dl) & & & & & & 249 & 260 \\
\hline $\mathrm{Na} \mathrm{K} \mathrm{Cl} \mathrm{Ca}(\mathrm{mmol} / \mathrm{l})$ & & $136 / 3.6 / 101 / 7.8$ & $139 / 4.3 / 109 / 8$ & $\mathrm{Na} 135$ & & & $139 / 4.0 / 100 / 7.9$ \\
\hline \multicolumn{8}{|l|}{ INMUNOLOGIA } \\
\hline PCR $(\mathrm{mg} / \mathrm{dl})$ & 19.7 & & & & & & 16.9 \\
\hline $\mathrm{HIV} / \mathrm{vdrl}$ & & & & & & $\mathrm{Neg} / \mathrm{Neg}$ & \\
\hline CMV IgG/IgM (U/L) & & $20.0(+) / 0.4(-)$ & & & & & \\
\hline Rubeola IgG/IgM (U/L) & & & & & & $(+) 89 /(-) 0.7$ & \\
\hline ToxoplasmalgG/IgM (U/L) & & & & & & $(-) 0.9 /(-) 0.3$ & \\
\hline HS1 IgG/IgM (U/L) & & & & & & $(-) 0.6 /(-) 0.4$ & \\
\hline VEBAR IgG /IgM (U/L) & & $1100 / 0.2$ & & & & & \\
\hline Ac ANA IFI & & & & $+1: 80$ nucleolar & & & \\
\hline Ac anti $C C P(U / L)$ & & & & & & 0.0 & \\
\hline
\end{tabular}




\begin{tabular}{|c|c|c|c|}
\hline $\lg \mathrm{G}, \operatorname{Ig} M \lg E \operatorname{Ig} A(\mathrm{mg} / \mathrm{d})$ & & & $\begin{array}{c}(+1) 2570 /(-1852 / \\
69 / 303\end{array}$ \\
\hline Hep A IgM & & $\mathrm{Neg}$ & \\
\hline Ag Hep B & & Neg & \\
\hline Ac anti $\mathrm{Hec}$ & & Neg & \\
\hline \multicolumn{4}{|l|}{ OTROS } \\
\hline CPS & & $\mathrm{Neg}$ & \\
\hline Inmunofenotipo: & & Sin malignidad & \\
\hline Hemocultivo & Negativo & & \\
\hline Urocultivo & $80,000 \mathrm{EA}$ & & \\
\hline \multicolumn{4}{|l|}{ ORINA } \\
\hline Leucocitos (LCSF) & & $35-45$ & \\
\hline Cilindros Granulosos (CCSF) & & De 3 a 5 & \\
\hline
\end{tabular}

\section{Tabla 2}

\section{Principales enfermedades que integran el síndrome hemofagocítico ${ }^{20}$.}

\begin{tabular}{|lllr|}
\hline Función defectuosa & Enfermedad & Gen & Proteína \\
\hline Activación celular S. & Linfoproteliferativo ligado a X & SH2D1A & SAP \\
\hline Exocitosis de granulos líticos (?) & S. Chédiak-Higashi & LYST & Lyst \\
\hline Polarización de gránulos líticos a la sinapsis inmune & S. Hermansky-Pudlak tipo 2 & AP381 & AP3B1 \\
\hline Anclaje de gránulos líticos a la membrana plasmática & S.Griscelli tipo 2 & Rab27a & Rab27a \\
\hline Destrucción célula diana & FHL-2 & PRF & Perforina \\
\hline Fusión de gránulos líticos a la membrana plasmática & FHL-3 & UNC13D & Munc 13-4 \\
\hline Fusión de gránulos líticos a la membrana plasmática (?) & FHL-4 & STX-11 & Sintaxina-11 \\
\hline Fusión de gránulos líticos a la membrana plasmática (?) & FHL-5 & STXBP2 & Munc 18-2 \\
\hline
\end{tabular}

FHL-2 : Linfohistiocitosis hemofagocítica familiar tipo 2; FHL-5: Linfohistiocitosis hemofagocítica familiar tipo 5; FHL-4: Linfohistiocitosis hemofagocítica familiar tipo 4; FHL-3: Linfohistiocitosis hemofagocítica familiar tipo 3.

(?) Mecanismo no aclarado 10, 16-18 


\section{Tabla 3}

\section{Principales elementos clínicos y para clínicos en los pacientes para realizar el diagnóstico ${ }^{24}$.}

\begin{tabular}{|c|c|c|c|c|c|}
\hline & Paciente $1 \mathrm{CHS}$ & Paciente 2 GS-2 & Paciente 3 GS- 2 & Paciente 4 FHL-3 & Paciente $5 \mathrm{FHL}-3$ \\
\hline Edad & 4 años & 17 meses & 10 años & 2 meses & 6 meses \\
\hline \multicolumn{6}{|l|}{ Sintomas } \\
\hline Fiebre & + & + & - & + & + \\
\hline Mal estado general & + & + & - & + & + \\
\hline Adenopatías & + & + & - & + & - \\
\hline Hepatoesplenomegalia & + & + & - & + & + \\
\hline Diátesis hemorrágica & + & $\cdot$ & - & - & - \\
\hline \multicolumn{6}{|l|}{ Fenotipo } \\
\hline Hipopigmentación piel & + & + & + & - & - \\
\hline Cabello gris plateado & + & + & + & $\cdot$ & $\cdot$ \\
\hline \multicolumn{6}{|l|}{ Datos analiticos } \\
\hline Pancitopenia & + & + & - & + & - \\
\hline Hipertrigliceridemia & + & + & - & + & - \\
\hline Hiperferritinemia & + & + & $\cdot$ & + & $\cdot$ \\
\hline \multicolumn{6}{|l|}{ Frotis sangre perif } \\
\hline Granulación anómala & + & $\cdot$ & - & - & $\cdot$ \\
\hline \multicolumn{6}{|l|}{ Médula ósea } \\
\hline Hemofagocitosis & - & + & - & + & - \\
\hline Granulación anómala & + & - & - & - & - \\
\hline \multicolumn{6}{|l|}{ Genética } \\
\hline Gen mutado & LYST & Rab27A & Rab27A & UNC13D & UNC13D \\
\hline Locus & $1942.1-42.2$ & $15 q 15-21.1$ & $15 q 15-21.1$ & $17 q 25.1$ & $17 q 25.1$ \\
\hline Síndrome & Chédiak-Higashi & Griscelli tipo 2 & Griscelli tipo 2 & FHL-3 & FHL-3 \\
\hline
\end{tabular}

